\title{
Effets comparés d'un aliment concentré et de l'aliment traditionnel des éleveurs sur les performances de croissance et économique des porcelets de race locale au Nord Cameroun
}

\author{
T.C. Keambou ${ }^{1 *}$ Y. Manjeli ${ }^{2}$ B.A. Hako ${ }^{2}$ \\ F. Meutchieye 2 J.C. Awono ${ }^{2}$
}

Mots-clés

Porcin - Jeune animal - Aliment composé pour animaux - Croissance Rentabilité - Cameroun.

\begin{abstract}
Résumé
L'effet d'un aliment concentré et de l'aliment traditionnel sur les performances pondérales du porc local du Nord Cameroun, et leur impact sur les revenus des éleveurs ont été comparés en milieu paysan pendant 75 jours. Au terme de l'essai, les porcelets alimentés avec le concentré ont présenté un poids de près de 60 p. 100 supérieur à celui des porcelets alimentés traditionnellement, soit un poids final respectivement de 26 et 16,1 kg. De même, les gains moyens quotidiens ont été de 206 et 74 g pour les porcelets soumis respectivement à l'aliment concentré et à l'aliment traditionnel. Sur le plan économique, la marge brute obtenue avec le concentré a été de 2 837,14 contre 950 francs CFA en moyenne avec les rations traditionnelles. Cette étude a montré qu'il serait possible d'améliorer la productivité des porcs de race locale du Nord Cameroun et ainsi le revenu des éleveurs en améliorant I'alimentation des animaux.
\end{abstract}

\section{INTRODUCTION}

Depuis plus d'une décennie au Cameroun, le rythme de la croissance démographique dépasse celui de la production agricole en général et des productions animales en particulier. L'une des conséquences est reportée sur la consommation journalière d'un Camerounais en protéines animales qui est seulement de 11,2 g, soit très en dessous des $32 \mathrm{~g}$ recommandés par individu par jour (6). La solution au problème d'apport alimentaire en protéine animale au Cameroun, qui est également le problème de la majorité des pays en développement, pourrait provenir du développement prioritaire des espèces domestiques, comme le porc et la volaille, à cycle court et dont l'élevage est facile.

\footnotetext{
1. Department of Plant and Animal Sciences, Faculty of Science, University of Buea, PO Box 63, Buea, Cameroon.

2. Département des productions animales, faculté d'Agronomie et des Sciences agricoles, Université de Dschang, Dschang, Cameroun.

* Auteur pour la correspondance

E-mail : christike2002@yahoo.fr
}

Dans la province du Nord Cameroun, l'élevage du porc est une activité en plein essor. Il repose sur des génotypes locaux et une conduite alimentaire essentiellement basée sur l'utilisation des ressources naturelles. Il est favorisé par la présence de races rustiques et une demande sans cesse croissante de viande de porc dans la partie méridionale du Cameroun (12). L'élevage de porcs dans le Nord Cameroun se caractérise par une alternance de périodes d'abondance en saison des pluies et de disette en saison sèche lorsque les ressources naturelles deviennent rares et que toutes les espèces domestiques doivent partager la faible production de sous-produits agricoles et agro-industriels. L'échec des premières tentatives empiriques de supplémentation des animaux pendant le période de disette a fait apparaître la nécessité de déterminer le potentiel de croissance du porc local, base scientifique indispensable à la mise en place de toute alternative de production.

Dans cette optique, cette étude a eu pour objectif principal de contribuer à l'amélioration de la productivité des porcs de race locale du Cameroun par une meilleure alimentation. Plus spécifiquement, les auteurs ont comparé la réponse d'une alimentation par rapport à celle traditionnellement pratiquée par les éleveurs sur le gain moyen quotidien, le poids final des animaux et la marge brute. 


\section{MATERIEL ET METHODES}

\section{Milieu d'étude}

L'essai à été conduit à Garoua, dans la province du Nord Cameroun (entre $7^{\circ}$ et $10^{\circ} 20^{\prime}$ de lat. $\mathrm{N}$, et $11^{\circ} 20^{\prime}$ et $15^{\circ} 10^{\prime}$ de long. E). Le climat est chaud et sec avec des températures moyennes annuelles de $31{ }^{\circ} \mathrm{C}$. Les températures varient entre 17 et $45^{\circ} \mathrm{C}$, et l'humidité relative est de 55 p. 100 en moyenne pour une pluviométrie de 800 à $1500 \mathrm{~mm}$ par an. La végétation est du type savane arborée ou forêt d'altitude. La population est à 90 p. 100 islamique. L'élevage des bovins et des petits ruminants y est prédominant ; celui du porc est en partie lié aux migrations récentes des populations païennes ou chrétiennes de toutes les régions du Cameroun, et surtout à l'absence de la peste porcine africaine dans cette partie du pays.

\section{Animaux}

Sept élevages ont été choisis sur la base de la disponibilité des éleveurs, l'existence d'une porcherie ayant des porcelets permettant de maintenir les animaux en claustration. Un effectif total de 86 porcelets tout-venant, âgés de quatre à cinq mois, a été recensé. Dans chaque élevage, le système de conduite, le poids et le sexe des animaux ont été notés. L'identification des porcelets a été effectuée à l'aide d'entailles aux oreilles. Dans chaque ferme, les porcelets ont été divisés en deux lots de même effectif et élevés en groupe. Dans le lot $\mathrm{C}$, ils ont été maintenus en claustration permanente pendant toute la durée de l'essai et ont reçu le concentré. Dans le lot T, ils ont été élevés selon les pratiques habituelles des éleveurs, avec divagation temporaire. Les soins vétérinaires de tous les animaux au début de l'essai se sont limités à un déparasitage interne (tétramisole injectable : $0,1 \mathrm{ml} / \mathrm{kg}$ de poids vif) et externe par pulvérisation d'Acar-dip $(8,17)$.

Dans chaque ferme, deux types de rations ont été distribuées : la ration $\mathrm{C}$ ou aliment concentré (tableau I), fabriqué en deux séquences et stocké dans un magasin sec à température ambiante, a été distribuée à volonté.

La ration $\mathrm{T}$ a été constituée des ingrédients habituellement utilisés par les éleveurs. Sa composition (tableau II) et la fréquence de distribution ont varié en fonction des fermes.

\section{Collecte des données}

Les porcelets ont été pesés au début de l'essai, puis tous les quinze jours dans la matinée avant la distribution de la ration journalière. La consommation alimentaire a été obtenue en faisant la différence entre les quantités d'aliment distribuées et les refus constatés. Les dépenses concernant l'aliment ont été obtenues à partir des prix pratiqués sur le marché de Garoua.

La marge brute a été calculée à partir de la différence entre la vente des animaux et les charges variables, selon les formules suivantes : Marge brute $=$ vente des animaux - charges variables Vente des animaux $=\mathrm{Q} \times \mathrm{P}$

\section{Tableau I}

Formulation de la ration et composition chimique du concentré

\begin{tabular}{lc} 
Ingrédients & Proportions $\mathbf{( \% )}$ \\
\hline Maïs & 40,0 \\
Son de riz & 30,0 \\
Tourteau de coton & 25,0 \\
Calcaire & 2,5 \\
Complément minéral et vitaminé & 2,0 \\
Sel de cuisine & 0,5 \\
Total & $\mathbf{1 0 0}$ \\
& \\
Caractéristiques & \\
& \\
Matière sèche & \\
Protéines brutes & 92,0 \\
Cellulose brute & 17,6 \\
Lysine & 10,0 \\
Méthionine & 2,0 \\
Calcium & 1,3 \\
Phosphate & 1,2 \\
Energie métabolisable (kcal/kg) & 0,8
\end{tabular}

\section{Tableau II}

Composition et fréquence de distribution des différentes rations utilisées par les éleveurs des porcs de race locale au Nord Cameroun

Ration

\section{Numéro des élevages}

\begin{tabular}{|c|c|c|c|c|c|c|c|}
\hline & 1 & 2 & 3 & 4 & 5 & 6 & 7 \\
\hline Drêche de bil-bil (bière artisanale de sorgho) & ++ & + & ++ & ++ & + & ++ & + \\
\hline Drêche de brasserie & - & - & - & - & + & - & + \\
\hline Bouillie d’arki (alcool artisanal) & - & - & + & - & - & - & - \\
\hline Déchet de cuisine & + & - & ++ & + & ++ & - & - \\
\hline Son de maïs & ++ & ++ & & ++ & & ++ & + \\
\hline Herbes & & + & ++ & & ++ & ++ & ++ \\
\hline Sel de cuisine & ++ & - & - & - & - & - & + \\
\hline Tourteau de coton & + & + & - & - & - & - & - \\
\hline Lait maternel & - & - & - & - & + & - & - \\
\hline Complément minéral et vitaminé & - & + & - & - & - & - & + \\
\hline Coques et amandes & + & - & - & - & - & - & - \\
\hline
\end{tabular}

++ Distribution régulière ; + distribution irrégulière ; - non distribué 
où $\mathrm{Q}$ est le poids des animaux à la vente $(\mathrm{kg}), \mathrm{P}$ le prix du kilogramme de porc vif sur le marché (FCFA $/ \mathrm{kg}$ ).

Les charges variables ont concerné essentiellement le coût des aliments, car ce type d'élevage est pratiqué au sein de certaines exploitations familiales de manière extensive avec un abri sommaire, la divagation des animaux et la rareté des soins.

\section{Analyse des données}

Les données collectées dans les différents élevages et pour les différents traitements ont été soumises à l'analyse de la variance (SAS) pour la comparaison des performances pondérales en fonction de la durée d'engraissement. Le modèle statistique utilisé a été le suivant :

$\mathrm{Y}_{\mathrm{ijkl}}=\mu+\mathrm{S}_{\mathrm{i}}+\mathrm{E}_{\mathrm{j}}+\mathrm{T}_{\mathrm{k}}+(\mathrm{TxE})_{\mathrm{jk}}+\mathrm{e}_{\mathrm{ijkl}}$

où $Y_{i j k l}$ est la performance de l'animal, $\mu$ la performance moyenne générale, $S_{i}$ l'effet du sexe, $E_{j}$ l'effet de l'élevage, $T_{k}$ l'effet de la ration, $(\mathrm{TxE})_{\mathrm{jk}}$ l'effet de l'interaction ration $\mathrm{x}$ élevage, $\mathrm{e}_{\mathrm{ijkl}}$ l'erreur résiduelle.

Le test de Duncan a été utilisé pour la séparation des moyennes chaque fois que les différences ont été significatives.

\section{RESULTATS ET DISCUSSION}

Le tableau II présente la composition et la fréquence de distribution des différentes rations alimentaires utilisées par les éleveurs de porcs de race locale au Nord Cameroun. Il montre aussi qu'une gamme variée de sous-produits agricoles et agro-industriels entre dans la composition des aliments des porcs locaux dans cette partie du pays. Le sous-produit le plus utilisé dans les fermes était la drêche de bil-bil (bière artisanale de sorgho), suivi du son de maïs et des herbes, puis des déchets de cuisine. Les coques et amandes, et la bouillie d'arki (alcool artisanal) étaient les moins distribuées, de même qu'un seul éleveur donnait accès au lait maternel.

\section{Evolution du poids}

L'évolution du poids moyen en fonction des différents facteurs d'influence et de la durée d'engraissement est présentée dans le tableau III. Sur toute la durée de l'essai, le poids des porcelets locaux n'a pas été significativement influencé par le sexe des animaux, alors que le facteur élevage n'a affecté $(\mathrm{P}<0,05)$ ce paramètre que pour une durée d'engraissement de 75 jours. En revanche, la ration distribuée et l'interaction ration x élevage ont eu un effet très significatif sur le poids des porcelets locaux sur toute la période de l'essai. A période égale, les femelles ont été sensiblement plus lourdes que les mâles et le concentré a offert une croissance plus rapide que la ration habituellement distribuée par les éleveurs.

Au $75^{\mathrm{e}}$ jour d'engraissement, le poids moyen des porcelets est passé de 10,5 à $26 \mathrm{~kg}$ avec le concentré, et de 10,5 à $16,1 \mathrm{~kg}$ avec les rations traditionnelles, soit une augmentation de plus de 60 p. 100 du poids final des porcelets soumis à l'aliment concentré. Ces

\section{Tableau III}

Analyse de la variance et poids moyen des porcelets de race locale du Cameroun en fonction des facteurs d'influence et de la durée d'engraissement

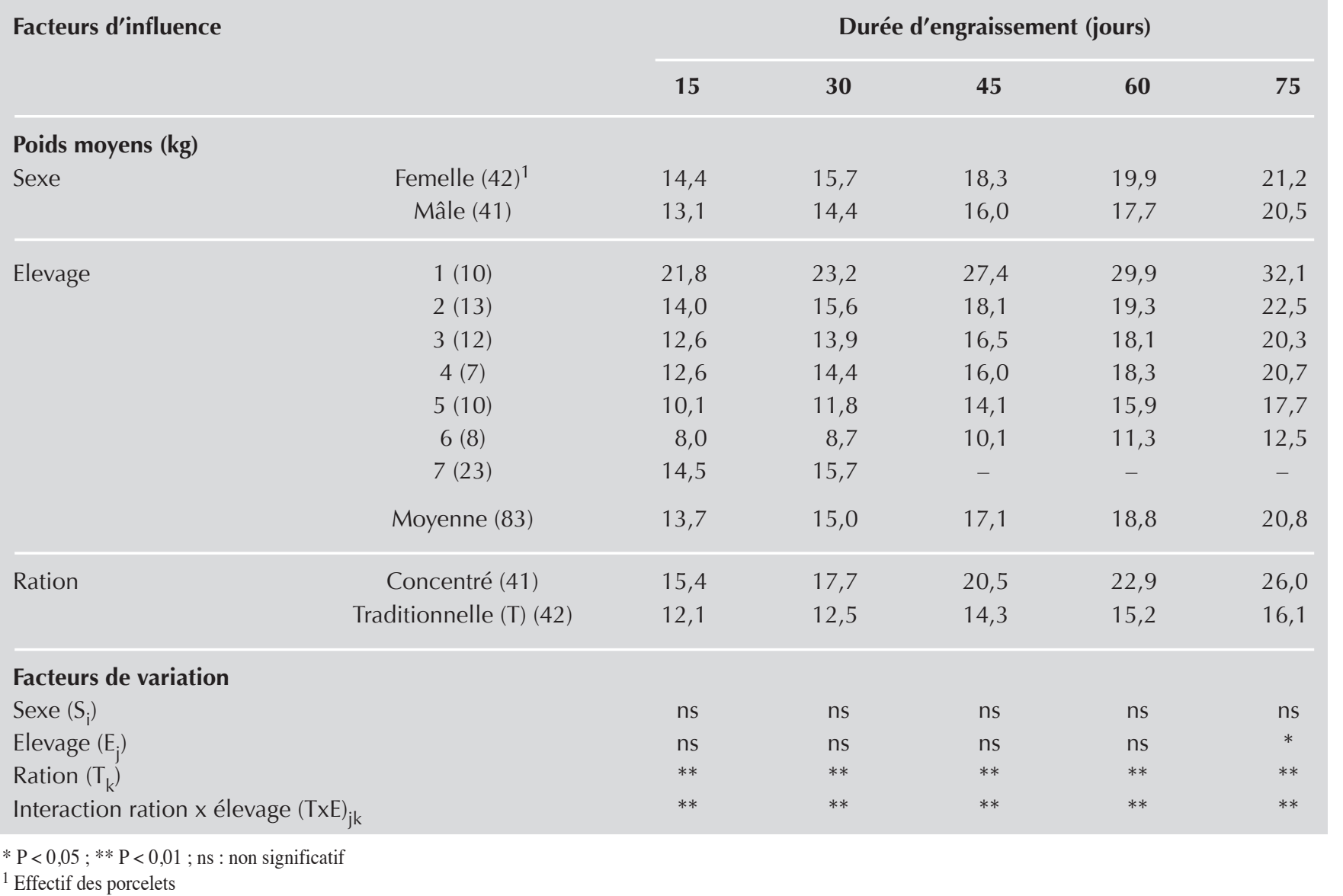


résultats corroborent ceux de Messi (11), Smith (16) et Soltner (18) sur l'effet d'une alimentation améliorée sur les performances des porcelets. Cependant, ces auteurs rapportent que le poids final des animaux dépend de plusieurs facteurs, comme la race, le poids initial (ou à la mise bas), le système d'alimentation et la durée d'engraissement. Quel que soit le système d'élevage, les performances de croissance des porcs de race locale du Cameroun sont très faibles, comparées à certaines souches européennes ou asiatiques. En outre, les systèmes de conduites inadaptés aggravent la situation. Les conditions climatiques (saison sèche très prolongée, température moyenne de $31^{\circ} \mathrm{C}$ avec des maxima à $45^{\circ} \mathrm{C}$, humidité relative moyenne de 55 p. 100) joueraient également sur ces performances ; Quiniou et coll. (13) rapportent que l'exposition des porcs à des températures élevées s'accompagne d'une chute des performances chez le porc sevré, en croissance ou en lactation.

\section{Gain moyen quotidien}

L'évolution du gain moyen quotidien (GMQ) en fonction des différents facteurs d'influence et de la durée d'engraissement est présentée dans le tableau IV. Le GMQ a été significativement influencé par le poids initial et l'effet du type de ration alimentaire tout au long de l'essai. En revanche, l'effet de l'interaction élevage x traitement n'a été significatif qu'à 15,30 et 75 jours, et l'effet élevage à 30,60 et 75 jours. Bien que Haer et de Vries (7) révèlent que les niveaux d'ingestion et des séquences alimentaires sont comparables chez les femelles et les mâles entiers, dans le cas de la présente étude, les femelles locales du Nord Cameroun ont consommé beaucoup plus que les mâles et avaient par conséquent un plus grand dépôt de tissu adipeux. Ceci serait à l'origine des gains de poids plus élevés chez les femelles tout au long de l'essai. La variation du GMQ observée entre les élevages entre les jours 0 et 75 de l'essai était due aux différences de la composition des aliments distribués et de la variabilité des quantités d'aliments journaliers disponibles. D'après les travaux de Schulze et coll. (14), et Mercat et Mormède (10) la consommation moyenne journalière est, parmi les critères de comportement alimentaire, la plus fortement corrélée aux performances de production et à la composition corporelle.

L'évolution du gain de poids des porcelets du Nord Cameroun nourris à l'aliment traditionnel et à l'aliment concentré ont présenté la même allure, ce qui indiquait probablement les effets du stress environnemental sur la productivité des animaux. Quelle qu'ait été la période, les porcelets nourris à l'aliment concentré ont présenté le gain de poids le plus élevé. Le GMQ enregistré avec le concentré lors de cet essai a été supérieur à celui du porc Bamiléké (11), de type Bakossi (4) et des élevages de types commerciaux des hautes terres de l'Ouest Cameroun (9). Il a été comparable à ceux enregistrés sur les porcs autochtones de Thaillande (5). En revanche, il a été inférieur à ceux enregistrés par Branckaert et Lecoq (1), Branckaert et coll. (2), Soltner (18), Douffissa (4), et Secondi et coll. (15). Chez le porc en croissance alimenté à volonté, l'ensemble des études met en évidence une diminution continue de la prise alimentaire et une baisse d'ingestion avec l'augmentation de la température ambiante, comme dans le cas du

\section{Tableau IV}

Evolution du gain moyen quotidien $(\mathrm{g})$ des porcelets de race locale en fonction des facteurs d'influence et de la durée d'engraissement dans le Nord Cameroun

Facteurs d'influence

Durée d'engraissement (jours)

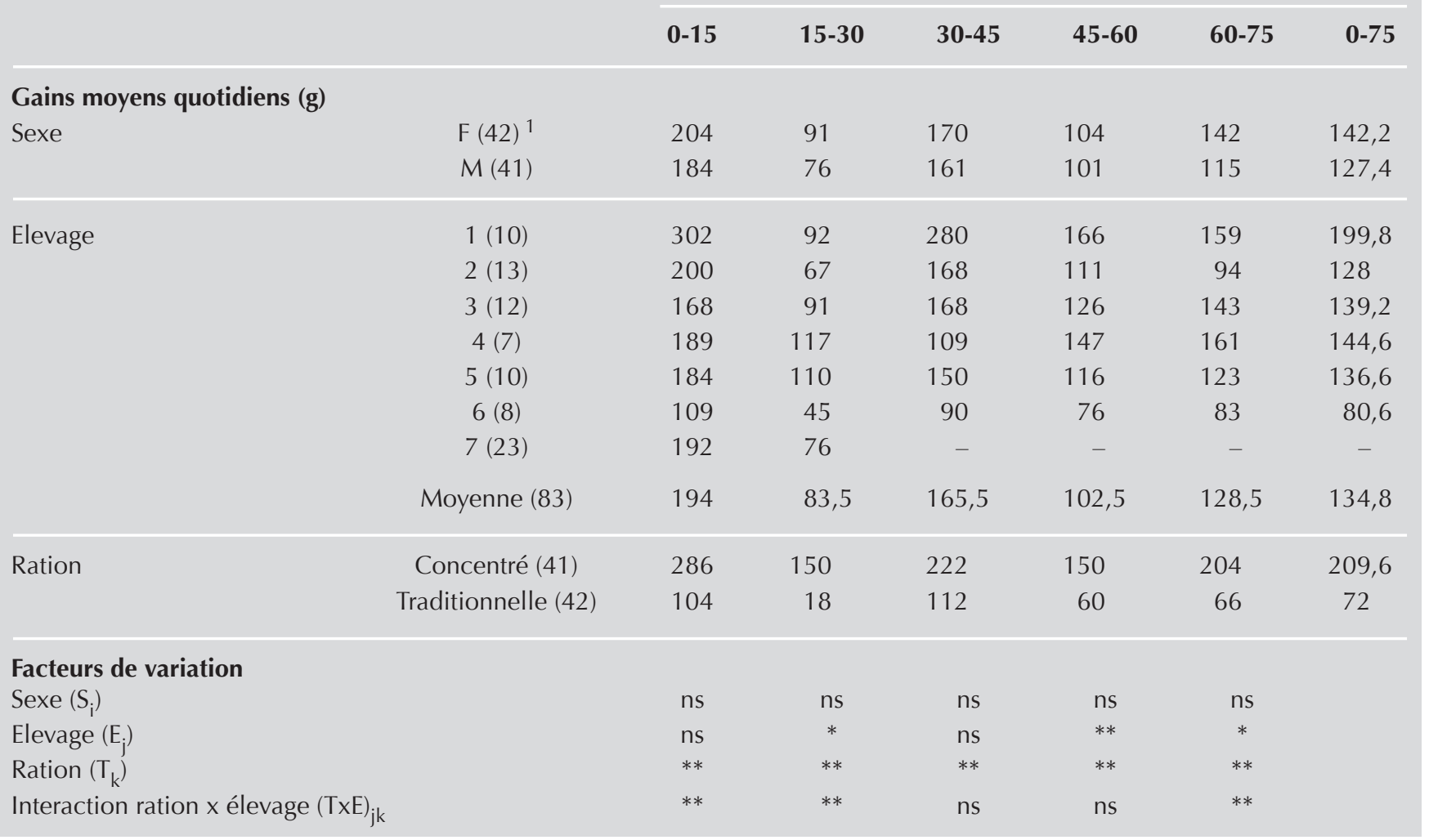

$* \mathrm{P}<0,05$; ** $\mathrm{P}<0,01 ;$ ns : non significatif, (n)

${ }^{1}$ Effectif des porcelets 
Nord Cameroun, expliquant également cette faible performance. La supériorité de la vitesse de croissance des animaux nourris à l'aliment concentré a été la conséquence du fait que les animaux ont été alimentés à volonté avec un régime équilibré (énergie métabolisable $=3000 \mathrm{kcal}$, taux de protéines $17,6 \mathrm{p}$. 100), contrairement à ceux des autres lots qui n'ont reçu qu'un aliment non mâ̂trisé en qualité et en quantité approximative. La prise spontanée d'aliment par un animal tend à coïncider, ou du moins à se rapprocher, de la quantité nécessaire à l'expression optimale de son potentiel de croissance et toute réduction de consommation est associée à une chute importante de la vitesse de croissance.

\section{Intérêt économique}

La figure 1 montre que, indépendamment de l'élevage, les charges, les ventes totales ainsi que la marge brute par animal ont été significativement plus élevées lorsque les animaux étaient alimentés avec le concentré, car ils atteignaient notamment un poids commercialisable plus rapidement que ceux qui recevaient la ration habituelle. La figure 2 montre les valeurs monétaires de ces paramètres économiques de l'élevage porcin en fonction du type d'aliment distribué dans le Nord Cameroun. Il a été cependant constaté que les prix de vente des porcelets ont été influencés par l'urgence des besoins de l'éleveur (scolarité, dot, maladie, funérailles ou autres), les fêtes civiles, religieuses, etc.

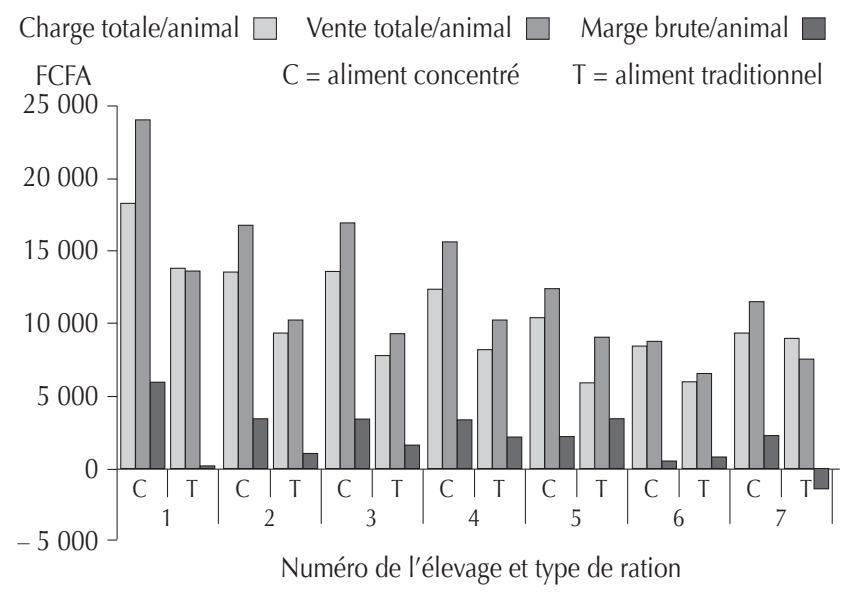

Figure 1 : charge totale moyenne, vente totale et marge brute (francs CFA) par animal en fonction des élevages et du type d'aliment.

\section{BIBLIOGRAPHIE}

1. BRANCKAERT R., LECOQ J., 1971. L'utilisation de la banane douce dans I'engraissement du porc (premiers résultats). Fruits, 26 : 15-20.

2. BRANCKAERT R., VALLERAND F., VINCENT J.C., 1973. La farine de cabosse de cacao dans l'alimentation du porc. Café Cacao Thé, 17 : 313320 .

3. COLEOU J., 1969. Préface. In : Lapierre O., Produits avicoles. Paris, France, Cereopa.

4. DOUFFISSA A., 1994. Livestock Korup project area pig husbandry. Yaoundé, Cameroun, ministère de l'Élevage et de la petite Industrie animale, p. 8-9. (Minepia-AGCD n 1)

5. FALVEY L., 1991. Recherche sur les porcins autochtones de Thaïlande. Revue mond. Zootech. (1) : 16-22.

6. FAO, 1995. Annuaire de production. Rome, Italie, FAO, 755 p.

\section{CONCLUSION}

L'aliment concentré a permis d'améliorer considérablement les performances pondérales des porcelets de race locale au Nord Cameroun. Les porcelets alimentés avec le concentré ont eu un poids final supérieur de plus de 60 p. 100 à celui des porcelets ayant reçu l'aliment traditionnel des éleveurs. De même, le GMQ des animaux soumis au concentré a pratiquement triplé.

Sur le plan économique, le prix du concentré a été nettement plus élevé que celui de la ration traditionnelle. La bonne marge économique brute de l'élevage porcin à l'aliment concentré dans le Nord Cameroun a souligné un potentiel économique important pour cet élevage, surtout en l'absence de peste porcine africaine dans cette partie du pays. Il n'a cependant pas été procédé à l'analyse de la carcasse. Bien que la viande de porc ne soit pas vendue au Cameroun en fonction de ses qualités physico-chimiques ou organoleptiques, la chair du porc local venant du Nord Cameroun est généralement plus appréciée par les consommateurs.

Le potentiel génétique des animaux locaux en terme de GMQ s'est situé entre 200 et $300 \mathrm{~g}$ sur la période considérée en excluant les périodes pendant lesquelles il y a eu un déficit de croissance. Les pratiques alimentaires traditionnelles n'ont pas permis d'atteindre ce potentiel.

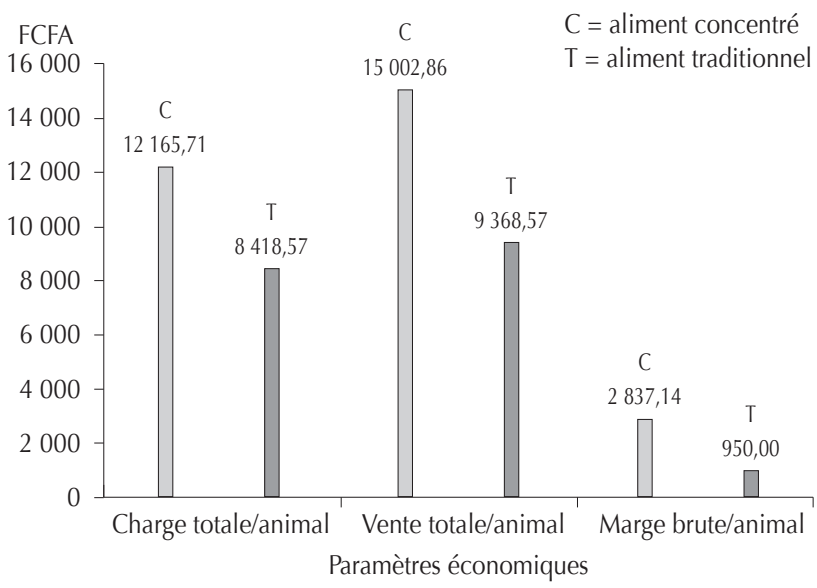

Figure 2 : valeur (francs CFA) des paramètres économiques de l'élevage porcin en fonction du type d'aliment distribué dans le Nord Cameroun.

7. HAER L.C.M., DE VRIES A.G., 1993. Effects of genotype and sex on the feed intake pattern of group-housed growing pigs. Livest. Prod. Sci., 36: 223-232.

8. LAPROVET, 1986. Spécialités vétérinaires. Notre Dame d'Oé, France, Laprovet, 77 p.

9. MANJELI Y., TCHOUMBOUE J., TEGUIA A., 1992. Quelques caractéristiques de l'élevage commercial de porc dans les hauts plateaux de l'Ouest Cameroun. Cameroon Bull. Anim. Prod. (1) : 24-31.

10. MERCAT M., MORMEDE P., 2002. Influences génétiques sur les processus d'adaptation et le comportement alimentaire chez le porc. Prod. Anim., 15 : 349-356.

11. MESSI J.M., 1982. Evaluation des performances de reproduction des types génétiques de porc à la station d'élevage de Kounden. Mém. fin étud., faculté d'Agronomie et des Sciences agricoles, Université de Dschang, Cameroun, 50 p. 
12. NJOYA A., AWA N.D., NGO TAMA A.C., 1996. Productivité des élevages porcins au Nord-Cameroun : Amélioration de la productivité porcine par l'alimentation et la prophylaxie. Garoua, Cameroun, IRVZ, 26 p.

13. QUINIOU N., RENAUDEAU D., COLLIN A., NOBLET J., 2000. Effets de l'exposition au chaud sur les caractéristiques de la prise alimentaire du porc à différents stades physiologiques. Prod. Anim., 13 : 233-245.

14. SCHULZE V., ROEHE R., LOOFT H., KALM E., 2001. Effects of continuous and periodic feeding by electronic feeders on accuracy of measuring feed intake information and their genetic association with growth performances. J. Anim. Breed. Genet., 118: 403-416.

15. SECONDI F., GANDEMER G., BONNEAU M., BERNARD E., SANTUCCI P.M., ECOLAN P., CASABLANCA F., 1996. Croissance, développement tissulaire et caractéristiques de la carcasse du porc corse. J. Rech. porcine Fr., 28 : 109-114.

16. SERRES H., 1989. Précis d'élevage du porc en zone tropicale. Maisons-Alfort, France, lemvt, 331 p. (Coll. Manuel et précis d'élevage)

17. SMITH A.J., 1991. Pig production. Trop. Anim. Health Prod., 12: $1-55$.

18. SOLTNER D., 1994. Alimentation des animaux domestiques, tomes 1 et 2. Sci. Tech. Agric. Sér. Zootech.

Accepté le 29.10.2011

\section{Summary}

Keambou T.C., Manjeli Y., Hako Touko B.A., Meutchieye F., Awono J.C. Compared effects of a concentrate and a traditional diet on growth and economic performances of young local-breed pigs in North Cameroon

The effects of a concentrate and a traditional diet on the weight performance of the local breed swine of North Cameroon, and their impact on farmers' incomes were compared on farm during 75 days. By the end of the trial, piglets fed the concentrate presented a weight about $60 \%$ higher than that of piglets fed the traditional diet, i.e. a final weight of 26 and $16.1 \mathrm{~kg}$, respectively. Similarly, the daily weight gain was 206 and $74 \mathrm{~g}$ in piglets that received the concentrate and the traditional diet, respectively. From an economic viewpoint, the gross margins obtained were 2837.14 CFA francs with 1 the concentrate and 950 CFAF with the traditional diet. This study showed that it is possible to improve the productivity of the local swine breed in North Cameroon and thus farmers' incomes by improving animal feed.

Keywords: Swine - Young animal - Compound feed Growth - Profitability - Cameroon.

\section{Resumen}

Keambou T.C., Manjeli Y., Hako Touko B.A., Meutchieye F., Awono J.C. Comparación de los efectos de un alimento concentrado y del alimento tradicional de los criadores sobre los rendimientos de crecimiento y económico de los lechones de raza local en el norte de Camerún

Durante 75 días, se comparó el efecto de un alimento concentrado y del alimento tradicional, sobre los rendimientos ponderales del cerdo local del norte de Camerún, así como el impacto en los ingresos de los criadores en un medio campesino. Al término del estudio, los lechones alimentados con alimento concentrado presentaron un peso casi $60 \%$ superior al de los lechones alimentados con el alimento tradicional, o sea 26 y 16,1 kg de peso final respectivamente. De la misma manera, las ganancias cotidianas promedio fueron de $206 \mathrm{~g} \mathrm{y}$ $74 \mathrm{~g}$ respectivamente para los lechones bajo alimento concentrado y aquellos que recibieron el alimento tradicional. Sobre el plano económico, el margen bruto obtenido con la ración concentrada fue de 2837,14 contra 950 francos CFA en promedio con las raciones tradicionales. Este estudio mostró que sería posible mejorar la productividad de los cerdos de raza local del norte de Camerún así como el ingreso de los criadores, mejorando la alimentación de los animales.

Palabras clave: Cerdo - Animal jóven - Pienso compuesto Crecimiento - Rentabilidad - Camerún. 